\title{
Computational sieving applied to some classical number-theoretic problems
}

\author{
Herman te Riele
}

\begin{abstract}
Many problems in computational number theory require the application of some sieve. Efficient implementation of these sieves on modern computers has extended our knowledge of these problems considerably. This is illustrated by three classical problems: the Goldbach conjecture, factoring large numbers, and computing the summatory function of the Möbius function.
\end{abstract}

1991 Mathematics Subject Classification: 11N35, 11P32, 11Y05, $11 Y 70$.

\section{Introduction}

In many problems in number theory like in prime counting, sieving plays a crucial role. Modern computers have made it possible to implement sieving in a very efficient way with the help of bit and vector operations, enabling us to extend the boundaries of our knowledge of these problems substantially. After discussing the arch-sieve of Eratosthenes and generalizations, we illustrate the progress in the past two decades with the following examples: the binary Goldbach conjecture which states that every even number $\geq 6$ can be expressed as a sum of two odd primes and the ternary Goldbach conjecture which states that every odd number $\geq 9$ can be expressed as a sum of three odd primes; factoring large numbers with sieve methods; computing the summatory function of the Möbius function.

\section{The sieve of Eratosthenes and its generalizations}

The best known sieve is the sieve of Eratosthenes (3rd century B.C.). It may be used to generate a table of prime numbers (and count them) up to some given bound $\mathrm{B}$ as follows. Among the integers in the interval $[2, B]$ all the multiples of 2 $(2 \times 2,3 \times 2, \ldots)$ are marked; next, all the multiples of the next smallest unmarked 
number are marked, and this is repeated until all the multiples of the primes $\leq \sqrt{B}$ have been marked. The numbers left unmarked are the primes $\leq B$.

One may also use this sieve to generate the primes in a given interval $[C, D]$ by sieving with all the primes $\leq \sqrt{D}$; this requires a small amount of additional work because for each sieving prime the smallest multiple of that prime in $[C, D]$ has to be found.

To illustrate this sieve, we generate the primes in $[100,140]$. We start by writing down the odd numbers in that interval (so the sieving with 2 has been done already) and we mark multiples of $3,5,7$, and 11 to get:

$$
\begin{aligned}
& \text { multiples of } 3: 101103 \underline{105} 107109 \underline{111} 113115 \underline{117} 119 \\
& 121 \underline{123} 125127 \underline{129} 131133 \underline{135} 137139 \\
& \text { multiples of } 5 \text { : } 101103 \underline{\underline{105}} 107109 \underline{111} 113 \underline{\underline{115}} \underline{117} 119 \\
& 121 \underline{123} \underline{\underline{125}} 127 \underline{129} 131133 \underline{\underline{\underline{135}}} 137139 \\
& \text { multiples of } 7 \text { : } 101103 \underline{\underline{105}} 107109 \underline{111} 113 \underline{\underline{115}} \underline{117} \underline{119} \\
& 121 \underline{123} \underline{\underline{\underline{125}}} 127 \underline{129} 131 \underline{133} \underline{\underline{135}} 137139 \\
& \text { multiples of } 11 \text { : } 101103 \underline{\underline{105}} 107109 \underline{111} 113 \underline{\underline{115}} \underline{117} \underline{119} \\
& \underline{121} \underline{123} \underline{\underline{\underline{125}}} 127 \underline{129} 131 \underline{133} \underline{\underline{135}} 137139
\end{aligned}
$$

The remaining, unmarked, numbers are prime because $139<13^{2}$. We notice that after the marking of multiples of some prime $\geq 3$, these numbers remain in the list. If instead one would drop these numbers, we obtain lucky numbers [25, sequence \# M2616], [11]; their density is higher than the density of the primes.

The sieve of Eratosthenes allows one to compute the number of primes $\leq x$, denoted by $\pi(x)$. According to the Prime Number Theorem, we have $\pi(x) \sim$ $x / \log x$, so with a sieve like that of Eratosthenes we cannot compute $\pi(x)$ with less than about $x / \log x$ operations. Various authors, starting with the astronomer Meissel in the 19th century, have studied faster methods. The best practical results have been obtained by Deléglise and Rivat [6], [5] who have computed various values of $\pi(x)$ for $x$ up to $10^{20}$ with an algorithm which has time complexity $\mathcal{O}\left(x^{2 / 3} / \log ^{2} x\right)$ and space complexity $\mathcal{O}\left(x^{1 / 3} \log ^{3} x\right)$.

An essential feature of the sieve of Eratosthenes is that it is concerned with counting the number of elements in a set that do not possess certain prescribed properties. This has been generalized in various directions: we mention here the books by Halberstam and Richert [12] and by Hooley [13] which treat numerous sieves which have the objective to estimate the number of unsifted elements in a set after the elements satisfying certain properties have been striked out.

One particular generalization is known as the Generalized Sieving Problem (GSP) [16]: suppose we are given

1. an interval $[C, D]$;

2. $\quad k$ moduli $m_{1}, m_{2}, \ldots, m_{k}$, all $>1$, relatively prime in pairs;

3. $k$ sets $\mathcal{R}_{i}=\left\{r_{i j} \mid 0 \leq r_{i j}<m_{i}\right\} \quad(i=1, \ldots, k)$ of acceptable residues. 
The question now is to determine all the integers $x \in[C, D]$ such that

$$
x\left(\bmod m_{i}\right) \in \mathcal{R}_{i} \quad \text { for } \quad i=1, \ldots, k .
$$

Let $m_{i}=p_{i}$, the $i$-th prime number. The instance $C=1, D=p_{k+1}^{2}$ for some positive integer $k$, and $\mathcal{R}_{i}=\left\{1,2, \ldots, p_{i}-1\right\}(i=1, \ldots, k)$, is the problem to find all the primes $<p_{k+1}^{2}$.

Another example of a GSP is the following. Let $f_{A}(x)$ denote the quadratic polynomial $x^{2}+x+A(x \in \mathbb{N} \cup\{0\}, A \in \mathbb{Z})$. Euler discovered that $f_{41}(x)$ is prime for forty consecutive values of $x$, namely, for $x=0,1, \ldots, 39$. Let $P_{A}(n)$ denote the number of prime values assumed by $f_{A}(x)$ for $0 \leq x \leq n$, so we have $P_{41}(39)=40 .{ }^{1)}$ Another example is $f_{27941}(x)$, discovered by N.G.W.H. Beeger in 1938 [2]: we have $P_{27941}(39)=30$, and $P_{27941}(1000000)=286128$, whereas $P_{41}(1000000)=261080$, so it seems that $P_{27941}(x)$ assumes more prime values than $P_{41}(x)$, albeit not for small $x$. The problem to find values of $A$ such that the density of prime values taken by $f_{A}(x)$ is high, can be formulated as a GSP. In order to find the polynomial $f_{27941}(x)$ Beeger computed all the positive integers $N<10^{6}$ of the form $8 t+3$ such that the Legendre symbol $(-N / q)=-1$ for all odd primes $q \leq 43$. A simple example is the problem of finding the least positive $X<8 \cdot 3 \cdot 5 \cdot 7=840$ such that

$$
\begin{aligned}
& X \equiv 3(\bmod 8) \\
& X \equiv 1(\bmod 3) \\
& X \equiv 2 \text { or } 3(\bmod 5), \\
& X \equiv 1,2, \text { or } 4(\bmod 7) .
\end{aligned}
$$

The solution is $X=43$.

\section{The Goldbach conjecture}

\subsection{The binary Goldbach conjecture}

The usual way to verify the binary Goldbach conjecture on a given interval $[A, B]$ is to mark those even $n \in[A, B]$ for which $n-p_{i}$ is prime, for $i=2,3, \ldots$, until all even $n \in[A, B]$ have been marked. This requires the availability of the primes in $[A, B]$ and a few small odd primes. As an example, we take $[A, B]=[100,138]$. Let

$$
\mathcal{P}=\{3,5,7,11,13,17,19\}
$$

and let

$$
\mathcal{Q}=\{89,97,101,103,107,109,113,127,131,137\}
$$

be the set of primes on the interval $[100,138]$ (and a few more). We start with writing down the even numbers in $[100,138]$ and mark (by underlining) those

1) It is known that $P_{A}(A-2)=A-1$ can only happen for $A=2,3,5,11,17,41$. 
belonging to the set $3+\mathcal{Q}$ :

$\frac{100}{120} 102 \underline{104} \underline{124} \frac{106}{126} 108 \underline{110} \underline{128} \underline{112} 114 \underline{116} 118$

Next, we mark those in $5+\mathcal{Q}$ to get:

$$
\frac{100}{120} \frac{102}{122} \frac{104}{124} \frac{106}{126} \frac{108}{128} \frac{110}{130} \frac{112}{\underline{132}} \frac{114}{134} \underline{116} \underline{136} \frac{118}{138}
$$

After marking, subsequently, the numbers which belong to $7+\mathcal{Q}, 11+\mathcal{Q}, 13+\mathcal{Q}$, we obtain:

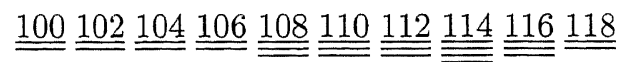

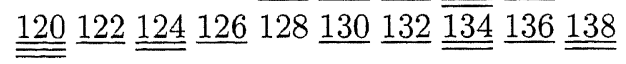

The remaining number, 128 , is somewhat "stubborn" since it is only marked with the set $19+\mathcal{Q} .^{2)}$

Notice that we have been building up the sum $\mathcal{P}+\mathcal{Q}$ to cover the even numbers in $[A, B]$. The set $\mathcal{P}$ contains a few small odd primes and the set $\mathcal{Q}$ contains the primes in $[A, B]$ (and a few more). An alternative approach is to choose for $\mathcal{P}$ the set of odd primes $\leq B-A$ and for $\mathcal{Q}$ a small set of large primes $<A$.

So, for $[A, B]=[100,138]$ we now start with the set $\mathcal{P}$ of odd primes $<38$ (and a few more):

$$
\mathcal{P}=\{3,5,7,11,13,17,19,23,29,31,37,41,43,47,53,59\}
$$

and

$$
\mathcal{Q}=\{79,83,89,97\}
$$

Again, we write down the even numbers in $[100,138]$ but we now mark those belonging to the set $\mathcal{P}+97$ to get:

$$
\underline{100} \underline{102} \underline{102} \underline{104} 106 \underline{108} \underline{124} \underline{126} \underline{128} \underline{130} 112 \underline{114} \underline{116} \underline{134} \underline{136} \underline{138}
$$

Doing the same for the sets $\mathcal{P}+89$ and $\mathcal{P}+83$ we obtain:

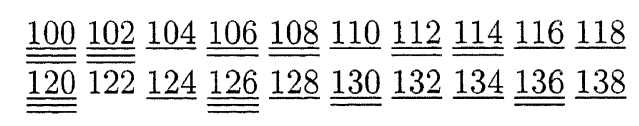

Finally, 122 is marked since it lies in the set $\mathcal{P}+79$.

The advantage of the second approach is that we have to generate the set of odd primes $\mathcal{P}$ below some given bound $B-A$ once; in addition, for each interval $[A, B]$ we have to find some primes smaller than but close to $A$. By keeping the length of the intervals $[A, B]$ fixed, we make optimal use of the set $\mathcal{P}$. In the first approach, one essentially has to generate all the primes in the intervals $[A, B]$ which we want to treat, and this is much more expensive than the work required in the second approach.

2) So $128-p$ is composite for $p=3,5,7,11,13,17$, but prime for $p=19$. 
With this idea of using a large set of "small" odd primes (namely the odd primes $<10^{9}$ ), and a small set of large primes near each interval $\left[A, A+10^{9}\right]$ to be checked, Deshouillers, Saouter, and the present author have verified the binary Goldbach conjecture up to $10^{14}$, partly on a Cray C90, partly on a cluster of workstations [9]. This extends similar work by Sinisalo up to $4 \times 10^{11}$ [24]. In addition, in [9] the binary Goldbach conjecture has been verified on the intervals $\left[10^{5 k}, 10^{5 k}+10^{8}\right]$, for $k=3,4, \ldots, 20$ and $\left[10^{10 k}, 10^{10 k}+10^{9}\right]$, for $k=20,21, \ldots, 30$. For each interval several hundred large primes close to the power of ten at the beginning of that interval were generated. Primality of these numbers was proved rigorously with the help of codes of François Morain $[17,1]$ and of Bosma and Van der Hulst [3].

\subsection{The ternary Goldbach conjecture}

The ternary Goldbach conjecture states that every odd number $\geq 9$ can be written as a sum of three primes. Recently, a proof of this conjecture was announced on the condition of the truth of the Generalized Riemann hypothesis [8]. In this proof use is made of the fact that the binary Goldbach conjecture is true for all even numbers $\leq 1.615 \times 10^{12}$, a result included in [9].

The (unconditional) truth of the ternary Goldbach conjecture up to $10^{20}$ was shown recently by Saouter [23], who, by using [24], constructed a sequence of about $2.5 \times 10^{\varangle}$ increasing prime numbers $q_{i}, 0 \leq i \leq P$ such that $q_{0}<4 \times 10^{11}$, $q_{i+1}-q_{i}<4 \times 10^{11}$ for all $0 \leq i \leq P-1$ and $q_{P}>10^{20}$.

\section{Factoring by sieving}

The problem of finding the prime factors of a given integer $N$ is old and well-known and many different factoring algorithms are known to day. The best algorithms for general numbers are based on finding integers $a$ and $b$ such that $a^{2} \equiv b^{2}(\bmod N)$, and ideas in this direction go back to Fermat, Legendre, Gauss and many others [10]. The quadratic sieve [19] and the number field sieve [14] are modern versions of such algorithms, and they have been used to factor the largest general numbers up to 130 decimal digits [4]. In these methods many $B$-smooth numbers have to be found, i.e., numbers whose prime factors are all $\leq B$. These $B$-smooth numbers are searched among numbers which are themselves values of polynomials and this makes it possible to use sieve methods for this purpose. We shall illustrate this with a simple example.

We want to factor $N$. Choose the quadratic polynomial

$$
\left.f(x)=\left(x+\left\lfloor N^{1 / 2}\right\rfloor\right)^{2}-N, \quad x=0, \pm 1, \pm 2, \ldots .3\right)
$$

Find $f(x)$-values that factor into primes less than some given bound $B$. If $f\left(x_{i}\right)$,

3) By $\lfloor x\rfloor$ we denote the largest integer $\leq x$. 
$i=1,2, \ldots$ are such values, we have

$$
\left(x_{i}+\left\lfloor N^{1 / 2}\right\rfloor\right)^{2} \equiv f\left(x_{i}\right)(\bmod N)
$$

and we try to find a subset of the $x_{i}$ 's such that the product of the corresponding $f\left(x_{i}\right)$-values is a square. If we succeed, we have found a congruence of the form $a^{2} \equiv b^{2}(\bmod N)$. We could try to find $B$-smooth $f(x)$-values by trial and error, but we can do much better by using the fact that any polynomial $f(x)$ enjoys the property that

$$
f(c) \equiv 0(\bmod d) \Longrightarrow f(c+k d) \equiv 0(\bmod d) \quad \text { for any } k \in \mathbb{Z} .
$$

Divisibility of $f(x)$ by some prime $p$ implies that

$$
\left(x+\left\lfloor N^{1 / 2}\right\rfloor\right)^{2} \equiv N(\bmod p)
$$

and this equation is solvable if $N$ is a quadratic residue of $p$, i.e., if the Legendre symbol $(N / p)=1$ (see, e.g., [22, Appendix 3]). For $N=1633, f(x)=$ $(x+40)^{2}-1633$, we find for the primes $\leq 7$ :

$$
\begin{aligned}
& f(x) \equiv 0(\bmod 2) \rightarrow x \equiv 1(\bmod 2) \\
& f(x) \equiv 0(\bmod 3) \rightarrow x \equiv 0,1(\bmod 3) \\
& f(x) \equiv 0(\bmod 5) \rightarrow \text { impossible } \\
& f(x) \equiv 0(\bmod 7) \rightarrow x \equiv-1,-2(\bmod 7) .
\end{aligned}
$$

These relations can be used to quickly sieve out 7 -smooth $f(x)$-values. A small table of $f(x)$-values looks as follows:

\begin{tabular}{rcc}
\hline$x$ & $f(x)$ & 7 -smooth \\
\hline-3 & $-264=-2^{3} \cdot 3 \cdot 11$ & no \\
-2 & $-189=-3^{3} \cdot 7$ & yes \\
-1 & $-112=-2^{4} \cdot 7$ & yes \\
0 & $-33=-3 \cdot 11$ & no \\
1 & $48=2^{4} \cdot 3$ & yes \\
2 & $131=$ prime & no \\
3 & $216=2^{3} \cdot 3^{3}$ & yes \\
\hline
\end{tabular}

To complete the algorithm, multiplying the above congruence relations corresponding to $x=-2,-1,1$, namely, $38^{2} \equiv-3^{3} \cdot 7,39^{2} \equiv-2^{4} \cdot 7$, and $41^{2} \equiv 2^{4} \cdot 3$, gives $(38 \cdot 39 \cdot 41)^{2} \equiv\left(2^{4} \cdot 3^{2} \cdot 7\right)^{2}(\bmod 1633), 341^{2} \equiv 1008^{2}(\bmod 1633)$, 1633 divides $341^{2}-1008^{2}, \operatorname{gcd}(341+1008,1633)=71$, and $1633=23 \cdot 71$.

\section{Computing arithmetic functions by sieving}

Another example where computational sieving plays a role is found in the problem of computing the values of an arithmetic function $f(n)$ for all $n$ in a given interval $[A, B]$, where $f(n)$ is a function of the prime factors of $n$. In addition, one may be interested in the behaviour of the summatory function $\sum_{1 \leq n \leq x} f(n)$. 
We will illustrate this with $f(n)=\mu(n)$, the Möbius function, defined by

$$
\mu(n)=\left\{\begin{array}{cl}
1, & n=1, \\
0, & \text { if } n \text { is divisible by a prime square } \\
(-1)^{k}, & \text { if } n \text { is the product of } k \text { distinct primes }
\end{array}\right.
$$

The function $M(x)=\sum_{1 \leq n \leq x} \mu(n)$ counts the difference between the number of squarefree positive integers $\leq x$ with an even number of prime factors and those with an odd number of prime factors. It plays an important role in analytic number theory. The boundedness of $M(x) / \sqrt{x}$ implies the truth of the Riemann hypothesis. Since Mertens, who conjectured that $M(x) / \sqrt{x}<1$, it has long been believed [20] that indeed $M(x) / \sqrt{x}$ is bounded, but nowadays one generally believes that this function is unbounded. This was supported by the disproof of Mertens' conjecture [18].

Lioen and Van de Lune [15] have found an efficient vectorized sieving algorithm for computing $\mu(n)$ for $n=1, \ldots, N$ :

$$
\begin{array}{ll}
\text { for } n=1 \text { to } N: & \mu(n)=1 \\
\text { for all } p \leq \sqrt{N}: & \text { (for all } n, p \mid n: \mu(n)=-p \cdot \mu(n)) \\
\text { for all } p \leq \sqrt{N}: & \text { (for all } \left.n, p^{2} \mid n: \mu(n)=0\right) \\
\text { for } n=1 \text { to } N: & (\text { if }|\mu(n)| \neq n \text { then } \mu(n)=-\mu(n)) \\
\text { for } n=1 \text { to } N: & \mu(n)=\operatorname{sign}(\mu(n))
\end{array}
$$

In Table 1 we illustrate this for $N=30$. The algorithm fills an array corresponding to $\mu(n)$ for $n=1, \ldots, 30$. The first column gives the indices $(1-30)$. The algorithm starts by initializing the array with 1 (column headed 1 ); next, every second element of this array is multiplied by -2 (column headed 2 ), every third element by -3 (column headed 3 ), and every fifth element by -5 (column headed 4 ). In the next three steps array elements with index divisible by the square of the primes 2,3 , and 5, respectively, are made zero (columns headed 5-7). In step 8 , an adjustment is made for array elements with index divisible by a prime $\geq 7$. In step 9 , each entry in the table is replaced by its sign-function, so the last column lists $\mu(n)$ for $n=1, \ldots, 30$.

Lioen and Van de Lune $[15,21]$ have applied this algorithm to compute $M(x)$ for all $x \leq 1.7889 \times 10^{13}$ on a Cray C90 vector computer, establishing the bounds

$$
-0.513<\frac{M(x)}{\sqrt{x}}<0.571
$$

The time complexity of this algorithm is $\mathcal{O}(x \log \log x)$ and the space complexity is $\mathcal{O}(x)$. Deléglise and Rivat [7] have given an algorithm to compute isolated values of $M(x)$ in time complexity $\mathcal{O}\left(x^{2 / 3}(\log \log x)^{1 / 3}\right)$ and space complexity $\mathcal{O}\left(x^{1 / 3}(\log \log x)^{2 / 3}\right)$. They list values of $M\left(a \times 10^{b}\right)$ for $a=1(1) 9$ and $b=10(1) 15$ and they give $M\left(10^{16}\right)=-3195437$. The corresponding $M(x) / \sqrt{x}$-bounds do not exceed those found by Lioen and Van de Lune. 


\begin{tabular}{|c|c|c|c|c|c|c|c|c|c|}
\hline$n$ & 1 & 2 & B & 1 & 5 & 6 & 7 & 8 & 9 \\
\hline 1 & 1 & & & & & & & & +1 \\
\hline 2 & 1 & -2 & & & & & & & -1 \\
\hline 3 & 1 & & -3 & & & & & & -1 \\
\hline 1 & 1 & -2 & & & 0 & & & & 0 \\
\hline$i$ & 1 & & & -5 & & & & & -1 \\
\hline 8 & 1 & -2 & $+t i$ & & & & & & +1 \\
\hline$i$ & 1 & & & & & & & -1 & -1 \\
\hline$x$ & 1 & -2 & & & 0 & & & & 0 \\
\hline 9 & 1 & & -3 & & & 0 & & & 0 \\
\hline 10 & 1 & -2 & & +10 & & & & & +1 \\
\hline 11 & 1 & & & & & & & -1 & -1 \\
\hline 12 & 1 & -2 & +6 & & 0 & & & & 0 \\
\hline 13 & 1 & & & & & & & -1 & -1 \\
\hline 14 & 1 & -2 & & & & & & +2 & +1 \\
\hline 15 & 1 & & -3 & +15 & & & & & +1 \\
\hline 16 & 1 & -2 & & & 0 & & & & 0 \\
\hline 17 & 1 & & & & & & & -1 & -1 \\
\hline 18 & 1 & -2 & +6 & & & 0 & & & 0 \\
\hline 19 & 1 & & & & & & & -1 & -1 \\
\hline 20 & 1 & -2 & & +10 & 0 & & & & 0 \\
\hline 21 & 1 & & -3 & & & & & +3 & +1 \\
\hline 22 & 1 & -2 & & & & & & +2 & +1 \\
\hline 23 & 1 & & & & & & & -1 & -1 \\
\hline 24 & 1 & -2 & +6 & & 0 & & & & 0 \\
\hline 25 & 1 & & & -5 & & & 0 & & 0 \\
\hline 26 & 1 & -2 & & & & & & +2 & +1 \\
\hline 27 & 1 & & -3 & & & 0 & & & 0 \\
\hline 28 & 1 & -2 & & & 0 & & & & 0 \\
\hline 29 & 1 & & & & & & & -1 & -1 \\
\hline 30 & 1 & -2 & +6 & -30 & & & & & -1 \\
\hline
\end{tabular}

Table 1. Vectorized computing of $\mu(n)$ for $n=1, \ldots, 30$

\section{References}

[1] Atkin, A.O.L., Morain. F., Elliptic curves and primality proving. Math. Comp. 61 (1993), 2968 .

[2] Beeger, N.G.W.H.. Report on some calculations of prime numbers. Nieuw Arch. Wisk. (2) 20 (1939). 48-50.

[3] Busma. W. van der Hulst. M.-P., Primality proving with cyclotomy. Ph.D. thesis. University of Amsterdam, December 1990.

[1] Cowie. J.. Dodson. B., Elkenbracht-Huizing, R.-M., Lenstra, A.K., Montgomery. P.L.. Zayer, J., A world wide number field sieve factoring record: on to 512 bits. In: Advances in cryptology - Asiacrypt 96 (ed. by K.J. Kim and T. Matsumoto; Lecture Notes in Comput. Sci. 1163), 382-394. Springer, Berlin 1996.

[5] Deléglise, M., Some new values of $\pi(x)$. Submitted for publication. 
[6] Deléglise, M., Rivat, J., Computing $\pi(x)$ : the Meissel, Lehmer, Lagarias, Miller, Odlyzko method. Math. Comp. 65 (1996), 235-245.

[7] - Computing the summation of the Möbius function. Experiment. Math. 5 (1996), 291-295.

[8] Deshouillers, J.-M., Effinger, G., te Riele, H., Zinoviev, D., A complete Vinogradov 3-primes theorem under the Riemann hypothesis. Electron. Res. Announc. Amer. Math. Soc. 3 (1997), 99-104.

URL: http://www.ams.org/journals/era/home-1997.html .

[9] Deshouillers, J.-M., te Riele, H.J.J., Saouter, Y., New experimental results concerning the Goldbach conjecture. In: Algorithmic number theory (ANTS-III, Portland, 1998) (ed. by J.P. Buhler; Lecture Notes in Comput. Sci. 1423), 204-215. Springer, Berlin 1998.

A preliminary version of this paper has appeared as CWI-Report MAS-R9804, March 1998, available as postscript file from ftp://ftp.cwi.nl/pub/CWIreports/MAS/MAS-R9804.ps.Z .

[10] Elkenbracht-Huizing, R.-M., Historical background of the number field sieve. Nieuw Arch. Wisk. (4) 14 (1996), 375-389.

[11] Gardiner, V., Lazarus, R., On certain sequences of integers defined by sieves. Math. Mag. 29 (1955), 117-122.

[12] Halberstam, H., Richert, H.-E., Sieve methods. Academic Press, London 1974.

[13] Hooley, C., Applications of sieve methods to the theory of numbers. Cambridge Univ. Press, Cambridge 1976.

[14] Lenstra, A.K., Lenstra, H.W., Jr., eds. The development of the number field sieve (Lecture Notes in Math. 1554). Springer, Berlin 1993.

[15] Lioen, W.M., van de Lune, J., Systematic computations on Mertens' conjecture and Dirichlet's divisor problem by vectorized sieving. In: From universal morphisms to megabytes: a Baayen space Odyssey (ed. by K. Apt, L. Schrijver, and N. Temme), 421-432. CWI, Amsterdam 1994.

[16] Lukes, R.F., Patterson, C.D., Williams, H.C., Numerical sieving devices: their history and some applications. Nieuw Arch. Wisk. (4) 13 (1995), 113-139.

[17] Morain, F., Courbes elliptiques et tests de primalité. Ph.D. thesis. L'Université Claude Bernard, Lyon I, September 1990. Introduction in French, body in English.

[18] Odlyzko, A.M., te Riele, H.J.J., Disproof of the Mertens conjecture. J. Reine Angew. Math. 357 (1985), 138-160.

[19] Pomerance, C., Analysis and comparison of some integer factoring algorithms. In: Computational methods in number theory, Part I (ed. by H.W. Lenstra, Jr. and R. Tijdeman; Math. Centre Tract 154), 89-139. Mathematisch Centrum, Amsterdam 1982.

[20] te Riele, H.J.J., On the history of the function $M(x) / \sqrt{x}$ since Stieltjes. In: Thomas Jan Stieltjes, Euvres complètes--Collected papers (ed. by G. van Dijk), vol. I, 69-79. Springer, Berlin 1993.

[21] te Riele, H.J.J., van de Lune, J., Computational number theory at CWI in 1970-1994. CWI Quarterly 7 (1994), 285-335. 
[22] Riesel, H., Prime numbers and computer methods for factorization, 2nd edn. Birkhäuser, Boston 1994.

[23] Saouter, Y., Checking the odd Goldbach conjecture up to $10^{20}$. Math. Comp. 67 (1998), 863-866.

[24] Sinisalo, M.K., Checking the Goldbach conjecture up to $4 \times 10^{11}$. Math. Comp. 61 (1993), 931-934.

[25] Sloane, N.J.A., Plouffe, S.. The encyclopedia of integer sequences. Academic Press, San Diego 1995. 